Annals of Pure and Applied Mathematics

Vol. 18, No. 2, 2018, 129-134

ISSN: 2279-087X (P), 2279-0888(online)

Published on 10 October 2018

www.researchmathsci.org

DOI: http://dx.doi.org/10.22457/apam.v18n2al

Annals of

Pure and Applied

Mathematics

\title{
On the Diophantine Equation $\left\{\left(q^{2}\right)^{n}\right\}^{x}+p^{y}=z^{2}$ where $q$ is any Prime Number and $p$ is an Odd Prime Number
}

\author{
Surya Prakash Gautam ${ }^{1}$, Hari Kishan ${ }^{2}$ and Satish Kumar ${ }^{3}$ \\ ${ }^{1}$ Department of Mathematics, DN College, Ch. Charan Singh University \\ Meerut - 250002, Meerut, India. \\ Corresponding author. Email: spgautam128@gmail.com \\ Email: ${ }^{2}$ harikishan10@ rediffmail.com; ${ }^{3}$ skg22967@gmail.com
}

Received 16 September 2018; accepted 9 October 2018

Abstract. In this paper, we have solved the Diophantine equation $\left\{\left(3^{2}\right)^{n}\right\}^{x}+p^{y}=z^{2}$ and $\left\{\left(\mathbf{5}^{\mathbf{2}}\right)^{\boldsymbol{n}}\right\}^{\boldsymbol{x}}+\boldsymbol{p}^{\boldsymbol{y}}=\mathbf{z}^{\mathbf{2}}$ where $\boldsymbol{n} \in \boldsymbol{Z}^{+}$and $\mathrm{p}$ is an odd prime. Also, we have discussed the generalization of $\left(4^{n}\right)^{x}+p^{y}=z^{2}$ to $\left\{\left(q^{2}\right)^{n}\right\}^{x}+p^{y}=z^{2}$, where $n \in Z^{+}$, q is any prime number and p is an odd prime number. Some solutions of these Diophantine equations have been obtained.

Keywords: Diophantine equations, Exponential Diophantine equations and Catalan's conjecture

\section{AMS Mathematics Subject Classification (2010): 11D61}

\section{Introduction}

Diophantine equations are central objects of number theory in mathematics with a vital importance in the field of Cryptography, Computer Science, Chemistry, Geometry and many more. According to Cao [4], the Diophantine equation $a^{x}+b^{y}=c^{z}$ has at most one solution for $z>1$. Suvarnamani et al. [9] proved that the Diophantine equation $4^{x}+7^{y}=z^{2}$ and $4^{x}+11^{y}=z^{2}$ have not any non-negative integer solution. Chatchaisthit [5] have presented that the Diophantine equation $4^{x}+p^{y}=z^{2}$ have the solutions of the form $(x, p, y, z) \in\{(2,3,2,5)\} \cup\left\{\left(r, 2^{r+1}+1,1,2^{r}+1\right): r \in N \cup\{0\}\right\} \cup$ $\left\{\left(r, 2,2 r+3,3.2^{r}\right): r \in N \cup\{0\}\right\}$ where $p$ is a prime number. Peker and Cenberci [8] worked on the Diophantine equation $\left(4^{n}\right)^{x}+p^{y}=z^{2}$ and the obtained solutions are $(x, y, z, p)=(1,2,5,3),(2,2,5,3)$ and $\left(k, 1,2^{n x}+1,2^{n x+1}\right)$, where $k$ is a non-negative integer and $p$ is an odd prime number , $n \in \mathrm{Z}^{+}$. Burshtein [1] discussed the conditions for the solution of Diophantine equation $p^{x}+q^{y}=z^{2}$ based on the various values of $\mathrm{p}$ and $\mathrm{q}$ where $\mathrm{p}, \mathrm{q}$ both are prime such that $p<q$ and differ by an even value $k$. Burshtein [2] discussed and found that the Diophantine equation $p^{x}+q^{y}=z^{2}$ has infinitely many solutions when $p=$ 2,3 and also demonstrated that if prime $p>3$ than the equation has a solution for each and every integer $x \geq 1$. Burshtein [3] discussed all the solutions to an open problem of Chotchaisthit on the Diophantine equation $2^{x}+p^{y}=z^{2}$ when $y=1$ and $p=7,13,29,37$, 
Surya Prakash Gautam, HariKishan and Satish Kumar

257. Kumar, Gupta and Kishan [6] solved the Diophantine equation $61^{\mathrm{x}}+67^{\mathrm{y}}=\mathrm{z}^{2}$ and $67^{\mathrm{x}}+73^{\mathrm{y}}=\mathrm{z}^{2}$ and proved that the equations have not any non-negative integer solution.

In this study, we discuss the Diophantine equation $\left\{\left(q^{2}\right)^{n}\right\}^{x}+p^{y}=z^{2}$ where $q$ is any prime number and $p$ is an odd prime number. We will use the Catalan's conjecture [7] and factor method to solve this Diophantine equation.

\section{Preliminary}

Lemma 2.1. Catalan's conjecture state that the only solution of the Diophantine equation $a^{x}-b^{y}=1$ is $(a, b, x, y)=(3,2,2,3)$ with $a>1, b>1, x>1$, and $y>1$.

Lemma 2.2. If $p$ is an odd prime $\& n \geq 2$ is integer, than $x^{2}-1=p^{n}$ has no solutions [7].

Lemma 2.3. The Diophantine equation $\left\{\left(2^{2}\right)^{n}\right\}^{x}+p^{y}=z^{2}$ has the solutions $(x, y, z, p)$ $=(1,2,5,3),(2,2,5,3)$ and $\left(k, 1,2^{n x}+1,2^{n x+1}\right)$ where $k$ is a non-negative integer [5].

\section{Main theorems}

Theorem 3.1. The Diophantine equation $\left\{\left(3^{2}\right)^{n}\right\}^{x}+p^{y}=z^{2}$, has the solution $(x, y, z$, $p)=\left(k, 1,3^{n k}+1,2.3^{n k}+1\right)$, where $p$ is an odd prime, $k \geq 0, n \in Z^{+}$and $x, y, z \in Z^{+} \cup\{0\}$.

Proof. We consider the Diophantine equation

$$
\left\{\left(3^{2}\right)^{n}\right\}^{x}+p^{y}=z^{2},
$$

where $n \in Z^{+}$and $x, y$ and $z$ are non-negative integers.

Now we discuss this problem in three cases.

Case 1. For $n=1$, equation (1) is given as

$$
\left\{\left(3^{2}\right)^{1}\right\}^{x}+p^{y}=z^{2}
$$

Or

$$
\left(3^{2}\right)^{x}+p^{y}=z^{2}
$$

Now for $y>0$,

$$
\left(3^{2}\right)^{x}+p^{y}=z^{2}
$$

Or

$$
z^{2}-\left(3^{2}\right)^{x}=\mathrm{p}^{y}
$$

Or

$$
\left(z-3^{x}\right)\left(z+3^{x}\right)=p^{y} .
$$

This implies that, $z-3^{x}=p^{v}, \quad z+3^{x}=p^{y-v}$, where $y>2 v$.

Now, we have

$$
p^{y-v}-p^{v}=2.3^{x}
$$

Or

$$
p^{v}\left(p^{y-2 v}-1\right)=2.3^{x}
$$


On the Diophantine Equation $\left\{\left(\boldsymbol{q}^{2}\right)^{n}\right\}^{x}+\boldsymbol{p}^{y}=\boldsymbol{z}^{2}$ where $q$ is any Prime Number and $p$ is an Odd Prime Number

For $v=0$,

$p^{y}-1=2.3^{x}$

Or

$p^{y}=2 \cdot 3^{x}+1$.

For $y=1$,

$p=2.3^{x}+1$

and

$z=3^{x}+1$.

Therefore we get $(x, y, z, p)=\left(k, 1,3^{k}+1,2.3^{k}+1\right)$, where $k$ is non-negative integer.

For $x=0$, the equation (1) can be written as

$$
\begin{array}{r}
\left(3^{2}\right)^{0}+p^{y}=z^{2} \\
z^{2}-p^{y}=1 .
\end{array}
$$

Or

By Lemma 2.1, it has no solution for $p$ an odd prime.

Now, for $y=0$, then the equation (2) can be written as

$$
z^{2}-3^{2 x}=1
$$

Therefore, it has no solution for $\mathrm{p}$ is an odd $\operatorname{prime}^{2}-1=3^{2 x}$ has no solution by Lemma 2.2.

Case 2. for $n=2$, equation (1) is given as

$$
\left\{\left(3^{2}\right)^{2}\right\}^{x}+p^{y}=z^{2}
$$

Or

$$
\left(3^{4}\right)^{x}+p^{y}=z^{2} \text {. }
$$

Now for $y>0$,

$$
\left(3^{4}\right)^{x}+p^{y}=z^{2}
$$

Or

$$
z^{2}-\left(3^{4}\right)^{x}=p^{y}
$$

Or

$$
\left(z-3^{2 x}\right)\left(z+3^{2 x}\right)=p^{y} .
$$

This implies that, $z-3^{2 x}=p^{v}, \quad \mathrm{z}+3^{2 x}=p^{y-v}$, where $y>2 v$

Now we have

$$
p^{y-v}-p^{v}=2.3^{2 x}
$$

Or

$$
p^{v}\left(p^{y-2 v}-1\right)=2.3^{2 x} \text {. }
$$

For $v=0$,

$$
p^{y}-1=2.3^{2 x}
$$

Or

$$
p^{y}=2.3^{2 x}+1 \text {. }
$$

For $y=1$, $p=2.3^{2 x}+1$ and $z=3^{2 x}+1$

Therefore we get $(x, y, z, p)=\left(k, 1,3^{2 k}+1,2.3^{2 k}+1\right)$, where $k$ is non-negative integer. For $y=0$ same as case 1 , and for $x=0$ same as case 1 .

Case 3. for all $n \in Z^{+}$equation (1) is given as 
Surya Prakash Gautam, HariKishan and Satish Kumar

$$
\left\{\left(3^{2}\right)^{n}\right\}^{x}+p^{y}=z^{2} .
$$

Now for $y>0$,

$$
\begin{gathered}
\left(3^{2 n}\right)^{x}+p^{y}=z^{2} \\
z^{2}-\left(3^{2 n}\right)^{x}=p^{y} \\
\left(z-3^{n x}\right)\left(z+3^{n x}\right)=p^{y} .
\end{gathered}
$$$$
\text { Or }
$$

Or

This implies that $z-3^{n x}=p^{v}, \quad z+3^{n x}=p^{y-v}$, where $y>2 v$

Now we have

$$
p^{y-v}-p^{v}=2.3^{n x}
$$

Or

For $v=0$,

$p^{v}\left(p^{y-2 v}-1\right)=2.3^{n x}$.

Or

$$
p^{y}-1=2.3^{n x}
$$

For $y=1, p=2.3^{n x}+1$ and $z=3^{n x}+1$

$$
p^{y}=2.3^{n x}+1 \text {. }
$$

Therefore we get $(x, y, z, p)=\left(k, 1,3^{n k}+1,2.3^{n k}+1\right)$, where $k$ is non- negative integer. For $y=0$ same as case 1 , and for $x=0$ same as case 1 .

Hence The Diophantine equation $\left\{\left(3^{2}\right)^{n}\right\}^{x}+p^{y}=z^{2}$, has the solution $(x, y, z, p)=(k, 1$, $\left.3^{n k}+1,2.3^{n k}+1\right)$, where $p$ is an odd prime, $k \geq 0, n \in Z^{+}$and $x, y, z \in Z^{+} \cup\{0\}$.

Theorem 3.2. The Diophantine equation $\left\{\left(5^{2}\right)^{n}\right\}^{x}+p^{y}=z^{2}$ has the solution $(x, y, z, p)$ $=\left(k, 1,5^{n k}+1,2.5^{n k}+1\right)$, where $p$ is an odd prime, $k \geq 0, n \in Z^{+}$and $x, y, z \in Z^{+} \cup\{0\}$.

Proof: The Diophantine equation

$$
\left\{\left(5^{2}\right)^{n}\right\}^{x}+p^{y}=z^{2}
$$

where $n \in Z^{+}$and $x, y, z \in Z^{+} \cup\{0\}$

Now for $y>0$,

Or

Or

This implies that,

We have

Or

For $v=0$,

Or

For $y=1$,

Thus we get $(x, y, z, p)=\left(k, 1,5^{n k}+1,2.5^{n k}+1\right)$, where $k$ is non- negative integer.

$$
\begin{gathered}
\left(5^{2 n}\right)^{x}+p^{y}=z^{2} \\
z^{2}-\left(5^{2 n}\right)^{x}=p^{y} \\
\left(z-5^{n x}\right)\left(z+5^{n x}\right)=p^{y} .
\end{gathered}
$$$$
z-5^{n x}=p^{v}, \quad z+5^{n x}=p^{y-v} \text {, where } y>2 v
$$$$
p^{y-v}-p^{v}=2.5^{n x}
$$$$
p^{v}\left(p^{y-2 v}-1\right)=2.5^{n x} \text {. }
$$$$
p^{y}-1=2.5^{n x}
$$$$
p^{y}=2.5^{n x}+1 \text {. }
$$

For $x=0$, $\left(5^{2 n}\right)^{0}+p^{y}=z^{2}$ 
On the Diophantine Equation $\left\{\left(\boldsymbol{q}^{2}\right)^{n}\right\}^{x}+\boldsymbol{p}^{y}=\boldsymbol{z}^{2}$ where $q$ is any Prime Number and $p$ is an Odd Prime Number

Or

$$
z^{2}-p^{y}=1
$$

By Lemma 2.1, it has no solution for $p$ is an odd prime.

Now, $y=0$, we have

$$
z^{2}-5^{2 n x}=1 .
$$

Therefore, $z^{2}-1=5^{2 n x}$ has no solution by Lemma 2.2.

Hence the Diophantine equation $\left\{\left(5^{2}\right)^{n}\right\}^{x}+p^{y}=z^{2}$ has the solution $(x, y, z, p)=(k, 1$, $\left.5^{n k}+1,2.5^{n k}+1\right)$, where $p$ is an odd prime, $k \geq 0, n \in Z^{+}$and $x, y, z \in Z^{+} \cup\{0\}$.

Theorem 3.3. The Diophantine equation $\left\{\left(q^{2}\right)^{n}\right\}^{x}+p^{y}=z^{2}$ has the solution $(x, y, z, p)$ $=\left(k, 1, \mathrm{q}^{n k}+1,2 . \mathrm{q}^{n k}+1\right)$, where $q$ is any prime number, $p$ is an odd prime, $k \geq 0, n \in Z^{+}$and $x, y, z \in Z^{+} \cup\{0\}$.

Proof: The Diophantine equation

$$
\left\{\left(q^{2}\right)^{n}\right\}^{x}+p^{y}=z^{2}
$$

where $n \in Z^{+}$and $x, y, z \in Z^{+} \cup\{0\}$

Now for $y>0$,

$$
\begin{gathered}
\left(q^{2 n}\right)^{x}+p^{y}=z^{2} \\
z^{2}-\left(q^{2 n}\right)^{x}=p^{y} \\
\left(z-q^{n x}\right)\left(z+q^{n x}\right)=p^{y} .
\end{gathered}
$$

Or

Or

This implies that,

$$
z-q^{n x}=p^{v}, \& z+q^{n x}=p^{y-v} \text {, where } y>2 v
$$

We have

$$
p^{y-v}-p^{v}=2 \cdot q^{n x}
$$

Or

$$
p^{v}\left(p^{y-2 v}-1\right)=2 \cdot q^{n x} .
$$

For $v=0$,

Or

$$
\begin{aligned}
& p^{y}-1=2 \cdot q^{n x} \\
& p^{y}=2 \cdot q^{n x}+1 .
\end{aligned}
$$

For $y=1$, $p=2 \cdot q^{n x}+1$ and $z=q^{n x}+1$.

Therefore we get $(x, y, z, p)=\left(k, 1, q^{n k}+1,2 \cdot q^{n k}+1\right)$, where $q$ is any prime number, $p$ is an odd prime and $k$ is non-negative integer.

For $x=0$,

$$
\left(q^{2 n}\right)^{0}+p^{y}=z^{2}
$$

Or

$$
z^{2}-p^{y}=1
$$

By Lemma 2.1, it has no solution for $p$ is an odd prime.

Now, for $y=0$, we have

$$
z^{2}-q^{2 n x}=1
$$

Therefore, $z^{2}-1=q^{2 n x}$ has no solution by Lemma 2.2 . 
Surya Prakash Gautam, HariKishan and Satish Kumar

Hence The Diophantine equation $\left\{\left(q^{2}\right)^{n}\right\}^{x}+p^{y}=z^{2}$ has the solution $(x, y, z, p)=(k, 1$, $\left.\mathrm{q}^{n k}+1,2 . \mathrm{q}^{n k}+1\right)$, where $q$ is any prime number, $p$ is an odd prime, $k \geq 0, n \in Z^{+}$ and $x, y, z \in Z^{+} \cup\{0\}$.

\section{Conclusion}

In this paper, we find out the solution $(x, y, z, p)=\left(k, 1, q^{n k}+1,2 . q^{n k}+1\right)$ of the Diophantine equation $\left\{\left(q^{2}\right)^{n}\right\}^{x}+p^{y}=z^{2}$, where $p$ is an odd prime and $k \geq 0$.

Acknowledgements. Surya Prakash Gautam indebted to the Human Resource Development Group Council of Scientific \& Industrial Research (HRDG-CSIR), for providing financial assistance in the term of Junior Research Fellowship (JRF). The authors are indebted to the referees for their valuable suggestions.

\section{REFERENCES}

1. N.Burshtein, On solutions of the Diophantine equation $p^{x}+q^{y}=z^{2}$, Annals of Pure and Applied Mathematics, 13 (1) (2017) 143 - 149.

2. N.Burshtein, On the Diophantine equation $p^{x}+q^{y}=z^{2}$, Annals of Pure and Applied Mathematics, 13 (2) (2017) 229 - 233.

3. N.Burshtein, All the Solutions to an Open Problem of S. Chotchaisthit on the Diophantine Equation $2^{x}+p^{y}=z^{2}$ when $y=1$ and $p=7,13,29,37,257$, Annals of Pure and Applied Mathematics, 16(1) (2018) 31-35.

4. Z.Cao, A note on the Diophantine equation $a^{x}+b^{y}=c^{z}$, Acta Arithmetica XCI,No1 (1999) 85-89.

5. S.Chotchaisthit, On the diophantine equation $4^{x}+p^{y}=z^{2}$, American Journal of Mathematics and Science, 1 (2012) 191-193.

6. S.Kumar, S.Gupta and H.Kishan, On the Non-Linear Diophantine Equation $61^{\mathrm{x}}+67^{\mathrm{y}}$ $=z^{2}$ and $67^{x}+73^{y}=z^{2}$, Annals of Pure and Applied Mathematics, 18 (2018) 91-94.

7. P.Mihailescu, primary cyclotomic units and a proof of Catalan's conjecture, J.Reine Angew. Math., 572 (2004) 167-195.

8. B.Peker and S.I.Cenberci, On the solutions of the equation $\left(4^{n}\right)^{x}+p^{y}=z^{2}$, arXiv:1202.2267 (2012) 2-5.

9. A.Suvarnamani, A.Singta and S.Chotchaisthit,On two diophantine equations $4^{x}+7^{y}=\mathrm{z}^{2}$ and $4^{x}+11^{y}=\mathrm{z}^{2}$, Science and Technology RMUTT Journal, 1 (2011) 25-28. 